\title{
Gross efficiency predicts a 6-min double-poling ergometer performance in recreational cross-country skiers
}

\author{
Niels Ørtenblad ${ }^{1,2} \cdot$ Kurt Jensen $^{1}$
}

Published online: 15 September 2017

(C) International Sports Engineering Association 2017

\begin{abstract}
The purpose of the study was to investigate which physiological parameters would most accurately predict a 6-min, all-out, double-poling (DP) performance in recreational cross-country skiers. Twelve male recreational cross-country skiers performed tests consisting of three series lasting $10 \mathrm{~s}$, one lasting $60 \mathrm{~s}$, plus a 6-min, all-out, DP performance test to estimate mean and peak power output. On a separate day, gross mechanical efficiency (GE) was estimated from a 10-min, submaximal, DP test and maximal oxygen consumption $\left(\mathrm{VO}_{2} \max \right)$ was estimated from an incremental treadmill running test. Power was measured after each stroke from the acceleration and deceleration of the flywheel that induced the friction on the ergometer. The power was shown to the skier on a small computer placed on the ergometer. A multivariable correlation analysis showed that GE most strongly predicted 6-min DP performance $(r=0.79)$ and interestingly, neither $\mathrm{DP} \mathrm{VO}_{2}$ max, nor treadmill-running $\mathrm{VO}_{2} \max$, correlated with 6-min DP performance. In conclusion, GE correlated most strongly with 6-min DP performance and $\mathrm{GE}$ at the ski ergometer was estimated to be $6.4 \pm 1.1 \%$. It is suggested that recreational cross-country skiers focus on skiing technique to improve gross mechanical efficiency during intense DP.
\end{abstract}

Niels Ørtenblad

nortenblad@health.sdu.dk

1 Department of Sports Science and Clinical Biomechanics, SDU Muscle Research Cluster (SMRC), University of Southern Denmark, Campusvej 55, 5230 Odense M, Denmark

2 Swedish Winter Sports Research Centre, Department of Health Sciences, Mid Sweden University, Östersund, Sweden
Keywords Ski-ergometer · Cross-country skiing · Doublepoling (DP) · Performance · Upper body exercise · Gross mechanical efficiency

\section{Introduction}

Cross-country skiing is practised on both a recreational and competitive level and has become a popular form of exercise. This is demonstrated by more than 50,000 skiers participating in Vasalop week every year, ranging from recreational to elite skiers. However, skiing on snow is not readily available, and certainly not on a regular basis, to a large percentage of the population having an interest in this form of exercise. Off-season training often occurs on dry land; this sometimes happens on roller skis and different ergometer devices have been developed for dry land use with the intention of simulating this form of exercise [1]. These skiing ergometers are used to simulate cross-country skiing and to train both endurance and high power outputs, and they are also used by athletes from other sports where a considerable work capacity is generated by the upper body (e.g. kayaking, swimming and rowing).

In 1967, Saltin and Astrand compared the maximal oxygen consumption $\left(\mathrm{VO}_{2} \max \right)$ among 133 of the best Swedish athletes across 19 different sports-including some who were among the world's best [2]. It was apparent from the study that the athletes with the highest $\mathrm{VO}_{2} \max$ values represented some of the most common endurance sports (i.e. cross-country skiing, running and bicycling). In conjunction with a high anaerobic threshold and well-developed movement economy, these three parameters are often referred to as the essential qualities for an athlete to possess in order to compete among the very best in endurance sports [3-5]. 
Several studies have reported that cross-country skiers have extraordinarily high $\mathrm{VO}_{2}$ max and anaerobic thresholds [2, 6, 7] and found that these parameters, together with movement economy, correlate very well with skiing performance $[6,8]$. It is of note that, although they are key determinants for performance, some of the parameters have been found to vary between cross-country skiers at the national and world-class levels [7]. Also, in untrained and recreational athletes, $\mathrm{VO}_{2} \max$ [9], anaerobic threshold [9, 10] and movement economy [11] are also known to determine performance in endurance sports.

Thus, while these three classic factors are key determinants of endurance performance, other physiological properties may influence skiing performance as well, especially in highly technical movements like cross-country skiing. In a group of sub-elite and highly trained recreational cross-country skiers, upper body (UB) peak poling power $(r=0.94)$, and mean 10-s $(r=0.93)$ and 60-s $(r=0.92)$ poling power, all correlated better with race speed in a $10-\mathrm{km}$ classic cross-country ski race than UB maximal oxygen uptake (UB $\mathrm{VO}_{2}$ peak, $r=0.88$ ) [12], indicating that explosive muscle properties (i.e. muscle power) and anaerobic capacity also play important roles in skiing performance. In line with this, training-induced improvements in upper body strength and power, and intense upper body interval training, have repeatedly been demonstrated to improve 3-10-min skiing performance [13-17]. Little is known about predictors of intense skiing performance in recreational cross-country skiers.

The purpose of this study was to investigate predictors for 6-min, all-out, DP performance in recreational crosscountry skiers. We hypothesise that gross mechanical efficiency (GE) will be a strong predictor of skiing performance, because it is likely to vary more in a group of recreational skiers than with elite skiers, due to the complex movements required in cross-country skiing. The physiological parameters analysed as predictors of performance are: 10-s DP power, 60-s DP power, GE, DP $\mathrm{VO}_{2}$ peak and $\mathrm{VO}_{2}$ max.

\section{Methods}

\subsection{Experimental outline}

Figure 1 shows an overview of the study design, which included three separate test days, with several days in between each test day. Two of the test days are identical (days $1+2$ ). The first day was used to familiarise the skiers with the procedure and test protocol, and the second day was used for the actual trial. On day 3, two submaximal tests and a maximal treadmill running test for the assessment of $\mathrm{VO}_{2}$ max were performed.

\subsection{Participants}

Twelve males participated in this study (age: $30 \pm 9$ years; height: $181 \pm 9 \mathrm{~cm}$; body mass: $81 \pm 7 \mathrm{~kg}$ ). All participants were recreational cross-country skiers with moderate technical skills in the sport. Several of the participants had participated in the Vasaloppet cross-country ski race more than once. Participants provided their informed consent and the study conforms to the Declaration of Helsinki and the ethical guidelines provided by the ethical committee of the Region of Southern Denmark, Denmark.

\subsection{Ski ergometer}

The ski ergometer used in this study was the air-resistance version of the ThoraxTrainer (ThoraxTrainer, Kokkedal, Denmark), which uses the same friction unit as the Dansprint kayak ergometer (Dansprint ApS, Hvidovre, Denmark). The flywheel is propelled by two ski poles through a string system attached to elastic bands inside the ergometer, and power is then measured directly in the ergometer flywheel. The elastic bands return the poles to their initial position after propulsion. The drag resistance of the ergometer is adjusted by altering the amount of air drawn into the flywheel. The lowest drag level is 1 and the highest is 10. Calibration of the ergometer was performed before each data collection according to the manufacturer guidelines.

\subsection{Test procedures}

The first two identical test days started with a 5-min warmup on the ski ergometer. This was followed by a series of all-out DP tests (Fig. 1); three 10-s tests at each drag level $[1,5$, and 10 , in this study only the tests at drag level 10 were used; (DP10)], one 60-s test at drag level 5 (DP60) and one 6-min test at drag level 1 (DP6). Resting periods between the tests were: 2 min between each of the 10 -s tests, $10 \mathrm{~min}$ between the last 10 -s test and the 60 -s test, and 45 min between the 60-s and the 6-min test. The DP10 with the highest mean power output was used for further analysis.

On the third test day, two 10-min submaximal DP tests were performed at drag level 1 in order to calculate the participant's gross mechanical efficiency (GE). The intensity of the two tests was targeted at 60 and $75 \%$ of the mean poling power measured during DP6 on day 2, and the participants were instructed to maintain this intensity as precisely as possible. The two tests were separated by only a very short break, during which time data from the ergometer were exported. The two submaximal DP tests were followed by a maximal running test performed on a treadmill (Woodway EXG 70, Waukesha, USA) with the 


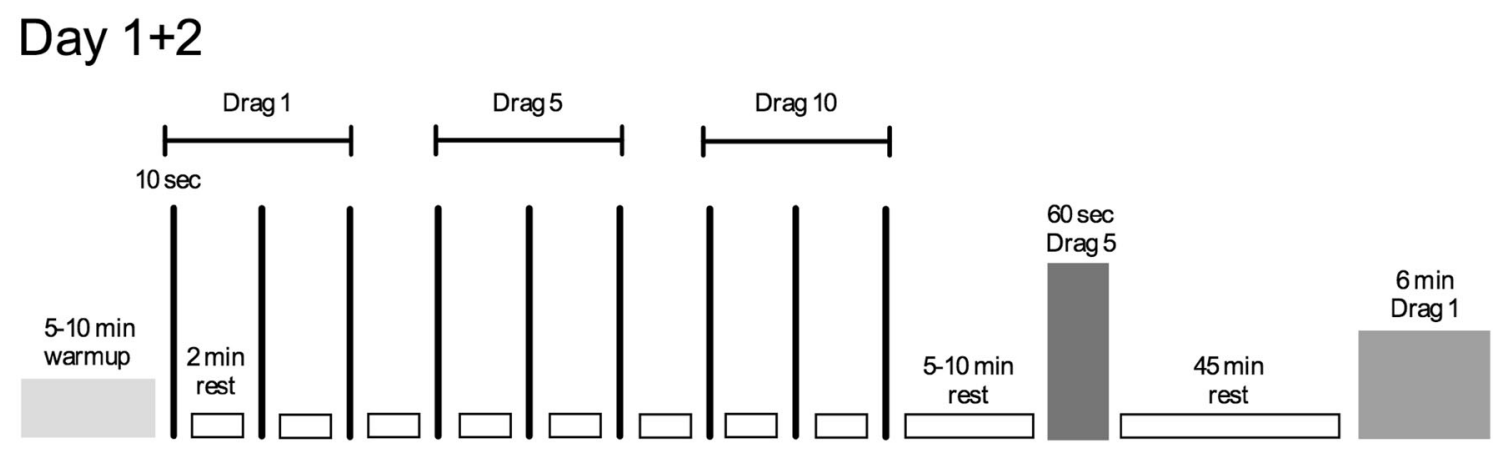

\section{Day 3}

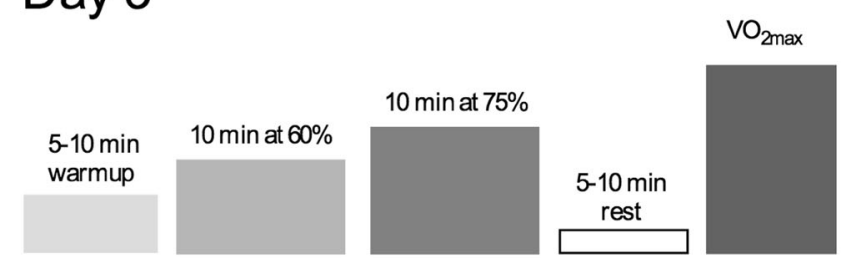

Fig. 1 Study overview. On days 1 and 2, five all-out double-poling (DP) tests were performed at resistance level (Res) 1 or 5. On day 3, two submaximal tests and a maximal treadmill test for the assessment of $\mathrm{VO}_{2}$ max were performed

purpose of measuring $\mathrm{VO}_{2}$ max. In this test, participants ran at $12 \mathrm{~km} / \mathrm{h}$ starting from a $0 \%$ incline with the inclination increasing by $2.5 \%$ every 3 min until exhaustion [18].

\subsection{Metabolic measurements and estimation of GE}

DP6 and the maximal running tests were performed with metabolic measurements [oxygen uptake $\left(\mathrm{VO}_{2}\right)$ (Oxycon Pro, Erich Jaeger GmbH, Hoechberg, Germany]; heart rate (HR) [Suunto T6c, Finland); blood lactate (BLa) (YSI 1500 Sport Lactate analyser, Yellow Springs Instrument Co., Yellow Springs, $\mathrm{OH})]$ and a subjective rating of perceived exertion (RPE) (6-20 Borg scale). The $\mathrm{VO}_{2}$ max during DP (DP $\mathrm{VO}_{2}$ peak) was defined as the highest 30-s mean $\mathrm{VO}_{2}$ value. The $\mathrm{VO}_{2}$ max during running was also defined as the highest 30-s mean $\mathrm{VO}_{2}$ value, and was regarded as valid if two of the following three parameters were met: (i) blood lactate (BLa) was above $8.0 \mathrm{mmol} / \mathrm{L}$, (ii) respiratory exchange ratio (RER) was above 1.10 , (iii) rating of perceived exertion (RPE) was 19 or 20. All participants had BLa and RER values above the required values.

$\mathrm{VO}_{2}$ was also measured during the last $4 \mathrm{~min}$ of each 10-min submaximal test. The GE at both intensities was then calculated as

$\mathrm{GE}=100 \times\left(P_{\text {ergo }} \times 60\right) /\left(20 \times \mathrm{VO}_{2}\right)$

where $P_{\text {ergo }}$ is the mean power measured in the ergometer flywheel in the last $4 \mathrm{~min}$ of each test and $\mathrm{VO}_{2}$ is the corresponding mean $\mathrm{VO}_{2}$ in the same period. Although not exact, a factor of $20 \mathrm{~kJ} / \mathrm{L}$ was chosen as mean value corresponding to an RER value of about 0.85 , or corresponding to a submaximal intensity of $70-80 \% \mathrm{VO}_{2} \max$. No significant differences were found in GE between the two submaximal tests, and so the GE presented is the mean value of the two tests pooled together.

\subsection{Statistical analysis}

Statistical significance was accepted at $p<0.05$. All values are given as mean \pm SD. To determine which parameters best predict 6-min all-out DP performance, a stepwise regression analysis was employed using the mean power output in DP6 from day 2 as the dependent variable. The independent variables and potential parameters predicting performance were as follows: DP10, DP60, DP $\mathrm{VO}_{2}$ peak (all from day 2), $\mathrm{VO}_{2}$ max, and GE.

\section{Results}

\subsection{Ski ergometer test performance and $\mathrm{VO}_{2}$}

One participant was excluded from the analysis due to a technical problem during the $\mathrm{VO}_{2}$ max measurement; hence, data from 11 participants were included in the regression analysis. Performance data from the ski ergometer tests were (in mean \pm SD); DP6 $100 \pm 22$ watts; DP10 $273 \pm 49$ watts and DP60 $187 \pm 29$ watts. The $\mathrm{VO}_{2} \max$ during $\mathrm{DP}, \mathrm{DP} \mathrm{VO}_{2}$ peak, was 
$4226 \pm 572 \mathrm{~mL} \mathrm{O} \mathrm{O}_{2} / \mathrm{min}$ and $\mathrm{VO}_{2}$ max during treadmill running was $4712 \pm 579 \mathrm{~mL} \mathrm{O} / \mathrm{min}$.

\subsection{Estimation of gross efficiency}

Gross efficiency was calculated from the $\mathrm{VO}_{2}$ measurements during the last $4 \mathrm{~min}$ of each of the 10-min submaximal tests and the $P_{\text {ergo }}$ measured directly in the ergometer flywheel, representing the total power to accelerate the fly wheel, excluding power loss from sledge and coil friction, as well as elastic resistance. The mean GE found was $6.4 \pm 1.1 \%$. This GE represents the total skiing ergometer work, excluding power loss from sledge and coil friction and elastic resistance.

\subsection{Predictors of 6-min performance}

DP10, DP60, and GE all correlated significantly with DP6 ( $r=0.65, r=0.74$, and $r=0.79$, respectively). There was no significant association between either $\mathrm{DP} \mathrm{VO}_{2}$ peak or $\mathrm{VO}_{2} \max (\mathrm{mL} / \mathrm{min})$ and DP6. The best predictor of DP6 was GE, which explained $57 \%$ of the variation $\left(r^{2}=0.57\right)$ in DP6. When $\mathrm{VO}_{2}$ peak was added to the regression model, it explained $78 \%\left(r^{2}=0.78\right)$ of the variation in DP6.

\section{Discussion}

The present study evaluated performance predictors for 6-min ski-ergometer DP performance in recreational crosscountry skiers. The main study findings were that 6-min all-out DP performance (DP6) was correlated with GE, DP10 and DP60, but not with DP $\mathrm{VO}_{2}$ peak or $\mathrm{VO}_{2}$ max.

\subsection{Performance predictors}

The high correlation between GE and 6-min DP performance was expected because the group investigated consisted of recreational skiers, who are expected to be more heterogenous in terms of skiing technique, and therefore GE, compared with elite cross-country skiers. An interesting result of this study was the absence of correlation between $\mathrm{DP} \mathrm{VO}_{2}$ peak and $\mathrm{VO}_{2}$ max with DP6. This indicates that some participants were not able to fully utilise $\mathrm{VO}_{2}$ to generate mechanical work during DP, possibly due to a less skilled DP technique. Moreover, significant correlations are more likely to be found when the variations are large in the independent variables. The between-participant variation in the parameters investigated as performance predictors was the largest for DP60 (56\% when expressed as the difference between the highest and lowest values as a percentage of the mean). GE had the second largest between-participant variation at 54\%, and $\mathrm{DP} \mathrm{VO}_{2}$ peak and $\mathrm{VO}_{2}$ max had smaller variations at 30 and $41 \%$. This might in part explain why $\mathrm{DP} \mathrm{VO}_{2}$ peak and $\mathrm{VO}_{2}$ max did not correlate with 6-min DP performance.

When compared with other upper and lower body tasks, the variation in GE found in this study and other cross-country skier studies performed on non-elite skiers appears greater. A study involving collegial cross-country skiers found a between-participant difference of $46 \%$ [19], which is not far from the variation found in the current group (54\%). In runners, the variation in running economy was much lower at $26 \%$ for recreational runners and $16 \%$ for well-trained runners, respectively [20,21], and in competitive female rowers, GE was found to be $19.7 \%$, with a between-participant difference reaching $36 \%$ (calculated from table and figure data) [22]. The large interindividual variation in GE found in our study population and supported by others may reflect the complexity of the DP movement compared to simpler motor skills like running. Besides GE, DP10 and DP60 were also correlated $(r=0.53$ and $r=0.74)$ with 6-min DP performance, which agrees with findings from Alsobrook and Heil showing that UB performance in 10- and 60-s maximal tests correlated more strongly with performance in a $10-\mathrm{km}$ massstart cross-country race than $\mathrm{DPVO}_{2}$ peak [12], proposing that the stronger correlations were likely due to underlying relationships between 10- and 60-s performance with GE. In this study, we did not find any such relationship between either DP10 $(r=0.15)$ or DP60 $(r=0.43, p=0.09)$ and GE. However, numerous training studies support the notion of UB performance as a better predictor of performance than $\mathrm{VO}_{2}$ peak. Thus, 9 weeks of explosive strength training in a group of relatively well-trained runners significantly improved running economy, which was presumed to result from neuromuscular changes [23], and similar findings were reported in a group of male cross-country skiers following 8 weeks of high intensity pulley-resistance training [13]. In the latter study, the authors put forward that the rate of force development and muscle power might be more important factors in improving movement economy than maximal strength itself, with better blood flow and lactate clearance within active muscles being underlying mechanisms that potentially explain the effect of resistance training [13].

\subsection{Gross efficiency in the ski ergometer}

The mean participant GE found was $6.4 \pm 1.1 \%$, which indicates a considerable power loss to the recoil system and/or friction compared to studies using other ergometer types. In a study using a Concept2 Ski ergometer (Morrisville, VT, USA), power was calculated directly in the flywheel, but using direct transformation by hands to the strings [24]. In that study, GE could be estimated from Fig. 5 to be $12 \%$, which is still low but closer to Ainegren 
et al., who calculated efficiency to be between 11.1 and $13.4 \%$ in diagonal skiing on a treadmill [25]. The deviation between the power measured using our ski ergometer adapted from kayaking and the GE measured on a treadmill or snow is consistent with the deviation in power reported by Holmberg and Nilsson using a ski ergometer adapted from rowing [1]. Thus, the efficiency in an ergometer could also be expressed including the aforementioned power loss from sledge friction, coil friction and elastic resistance, thereby increasing the value for efficiency. These findings suggest that when such ski ergometers are used in testing situations, a prior validation study is required to establish the relationship between ergometer-measured power and pole power for precise quantification of performance.

\section{Conclusion}

The main findings of this study were that GE correlated most strongly with 6-min DP performance and that neither $\mathrm{DP} \mathrm{VO}_{2}$ peak nor $\mathrm{VO}_{2}$ max correlated with 6-min DP performance. The present findings suggest that moderately trained crosscountry skiers should focus on improving their technique to increase their DP gross mechanical efficiency rather than their maximal oxygen uptake, if the intention is to improve DP cross-country skiing performance.

\section{References}

1. Holmberg H-C, Nilsson J (2008) Reliability and validity of a new double poling ergometer for cross-country skiers. J Sports Sci 26(2):171-179

2. Skattebo Ø, Hallén J, Rønnestad BR, Losnegard T (2016) Upper body heavy strength training does not affect performance in junior female cross-country skiers. Scand J Med Sci Sports 26(9):1007-1016

3. Bassett DR, Howley ET (2000) Limiting factors for maximum oxygen uptake and determinants of endurance performance. Med Sci Sports Ex 32(1):70-84

4. Joyner MJ (1991) Modeling: optimal marathon performance on the basis of physiological factors. J Appl Physiol 70(2):683-687

5. Joyner MJ, Coyle EF (2008) Endurance exercise performance: the physiology of champions. J Physiol 586(1):35-44

6. Larsson P, Olofsson P, Jakobsson E, Burlin L, Henriksson-Larsén K (2002) Physiological predictors of performance in crosscountry skiing from treadmill tests in male and female subjects. Scand J Med Sci Sports 12(6):347-353

7. Sandbakk O, Ettema G, Leirdal S, Jakobsen V, Holmberg H-C (2011) Analysis of a sprint ski race and associated laboratory determinants of world-class performance. Eur J Appl Physiol 111(6):947-957

8. Saltin B, Åstrand P-O (1967) Maximal oxygen uptake in athletes. J Appl Physiol 23(3):353-358

9. Stratton E, O'Brien B, Harvey J, Blitvich A, McNicol A, Janissen D, Paton C, Knez W (2008) Treadmill velocity best predicts 5000-m run performance. Int J Sports Med 30(01):40-45

10. Izquierdo-Gabarren M, Expósito RGDT, de Villarreal ESS, Izquierdo M (2010) Physiological factors to predict on traditional rowing performance. Eur J Appl Physiol 108(1):83-92

11. Tartaruga MP, Mota CB, Peyre-Tartaruga LA, Brisswalter J (2014) Scale model on performance prediction in recreational and elite endurance runners. Int j Sports Physiol Perf 9(4):650-655

12. Alsobrook NG, Heil DP (2009) Upper body power as a determinant of classical cross-country ski performance. Eur J Appl Physiol 105(4):633-641

13. Hoff J, Gran A, Helgerud J (2002) Maximal strength training improves aerobic endurance performance. Scand J Med Sci Sports 12(5):288-295

14. Losnegard T, Mikkelsen K, Rønnestad BR, Hallén J, Rud B, Raastad T (2011) The effect of heavy strength training on muscle mass and physical performance in elite cross country skiers. Scand J Med Sci Sports 21(3):389-401

15. Nesser TW, Chen S, Serfass RC, Gaskill SE (2004) Development of upper body power in junior cross-country skiers. J Strength Cond Res 18(1):63-71

16. Nilsson JE, Holmberg HC, Tveit P, Hallén J (2004) Effects of 20 -s and 180-s double poling interval training in cross-country skiers. Eur J Appl Physiol 92(1-2):121-127

17. Sandbakk Ø, Holmberg H-C, Leirdal S, Ettema G (2010) Metabolic rate and gross efficiency at high work rates in world class and national level sprint skiers. Eur J Appl Physiol 109(3):473-481

18. Balke B, Ware RW (1959) An experimental study of physical fitness of Air Force personnel. US Armed Forces Med J 10:675

19. Nakai A, Ito A (2011) Net efficiency of roller skiing with a diagonal stride. J Sports Sci 29(4):423-429

20. Heise GD, Martin PE (2001) Are variations in running economy in humans associated with ground reaction force characteristics? Eur J Appl Physiol 84(5):438-442

21. Heise GD, Morgan DW, Hough H, Craib M (1996) Relationships between running economy and temporal EMG characteristics of bi-articular leg muscles. Int J Sports Med 17(2):128-133

22. Hofmijster MJ, Van Soest AJ, De Koning JJ (2009) Gross efficiency during rowing is not affected by stroke rate. Med Sci Sports Ex 41(5):1088-1095

23. Paavolainen L, Hakkinen K, Hamalainen I, Nummela A, Rusko H (1999) Explosive-strength training improves 5-km running time by improving running economy and muscle power. J Appl Physiol 86(5):1527-1533

24. Hegge AM, Bucher E, Ettema G, Faude O, Holmberg HC, Sandbakk O (2016) Gender differences in power production, energetic capacity and efficiency of elite cross-country skiers during whole-body, upper-body, and arm poling. Eur J Appl Physiol 116(2):291-300

25. Ainegren M, Carlsson P, Tinnsten M, Laaksonen MS (2013) Skiing economy and efficiency in recreational and elite crosscountry skiers. J Strength Cond Res 27(5):1239-1252 